\title{
Giant Atrial Septal Artery Aneurysm with a Fistula to the Right Atrium
}

\author{
Bo Mei, MD, PhD,,$^{1,2}$ Guangjie He, BD, ${ }^{1,2}$ Xiong Tan, MD, ${ }^{1,2}$ Weitao Jin, MD, ${ }^{1,2}$ Yaxi Zhao, MD, ${ }^{3}$ \\ Yinglong Lai, $\mathrm{MD}^{1,2}$
}

${ }^{1}$ Department of Cardiovascular Surgery; ${ }^{2}$ Laboratory of Herat-Lung Transplantation; ${ }^{3}$ Department of Ultrasound, Affiliated Hospital of North Sichuan Medical College, Nanchong, Sichuan, China

\section{ABSTRACT}

An atrial septal artery aneurysm is a rare disease, especially accompanied with a fistula. During clinical practice, it is very important to clear the anatomical details of the coronary aneurysm before operation. In the current article, we report a giant atrial septal artery aneurysm originating from a branch of the RCA combined with a coronary right atrial fistula. The coronary artery aneurysm should be evaluated using multiple diagnostic and imaging modalities, such as echocardiography, coronary artery angiography, magnetic resonance imaging, and cardiac CT, especially three-dimensional reconstruction, which could help us to distinguish the physiological and anatomical characteristics of the CAA and fistula.

\section{INTRODUCTION}

An atrial septal artery aneurysm is a rare disease, especially accompanied with a fistula. Here, we report a giant atrial septal artery aneurysm originating from a branch of the RCA combined with a coronary right atrial fistula. The coronary artery aneurysm was evaluated using multiple diagnostic and imaging modalities.

\section{CASE PRESENTATION}

A 48-year-old female patient presented with a 6-year history of chest distress and palpitations after physical activity. On arrival, she had no symptoms of heart failure. Her blood pressure was $120 / 52 \mathrm{~mm} \mathrm{Hg}$, with a heart rate of 85 beats $/ \mathrm{min}$ and oxygen saturation of $99 \%$. Physical examination revealed a continuous grade III/VI murmur at the intersection of the left sternal area and the third and fourth intercostal spaces. Her breathing sounded normal and without respiratory distress. Twenty-four hour dynamic electrocardiography revealed paroxysmal atrial fibrillation. Echocardiography revealed the presence of a large right coronary artery (RCA) saccular aneurysm with a fistula to the right atrium (RA), severe tricuspid

Received fune 9, 2021; accepted fune 28, 2021.

Correspondence: Yinglong Lai, MD, Department of Cardiovascular Surgery, Affiliated Hospital of North Sichuan Medical College, 1st Maoyuan South Road, Nanchong, Sichuan 637000, China; +86-817-2598352; fax: +86-817-2222856 (e-mail laiyinglong2000@163.com).. insufficiency, and moderate mitral insufficiency (Figure 1A and 1B). Transesophageal echocardiography further revealed
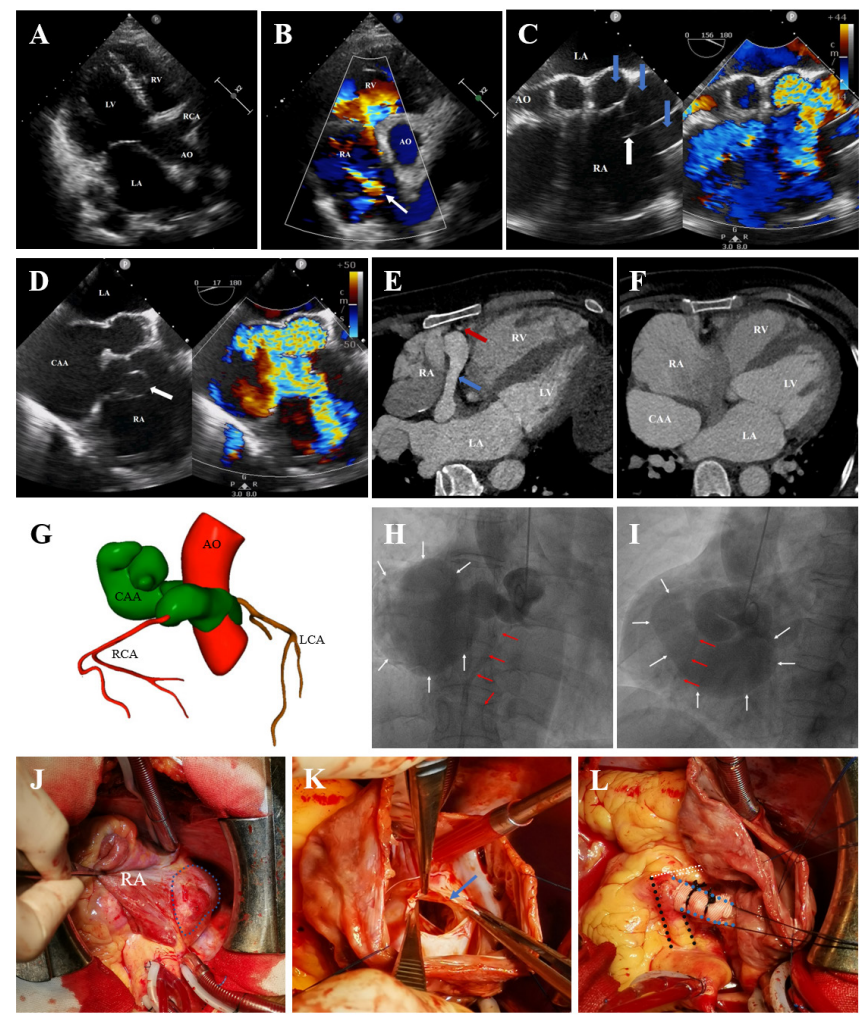

Figure 1. Multiple examination images. A-D) Echocardiography and transesophageal echocardiography revealed a large RCA aneurysm (blue arrow) with a fistula (white arrow) to the RA. E-F) Cardiac CT showed a giant CAA originating from an atrial branch of the RCA (red arrow, normal RCA; blue arrow, anomalous branch of the RCA). G) Three-dimensional reconstruction clearly showed a giant atrial septal artery aneurysm (green) with a fistula to the RA. H-I) Coronary angiography confirmed the CAA and the fistula (red arrow, normal RCA; white arrow, CAA). J-K) Surgical findings demonstrated that a giant CAA (blue circle) was located on the right lateral surface of the right atrioventricular sulcus and that the fistula was identified as a $10-\mathrm{mm}$ hole (blue arrow) at the lateral wall of the RA during surgery. L) the proximal atrial septal artery was knotted (black dots, proximal RCA dilatation; white dots, normal RCA; blue dots, proximal atrial branch of the RCA). For further images, please refer to Videos I-IV in the Data Supplement. RA, right atrium; RV, right ventricle; LA, left atrium; LV, left ventricle; $A O$, aorta; CAA, coronary artery aneurysm; RCA, right coronary artery; LCA, left coronary artery 


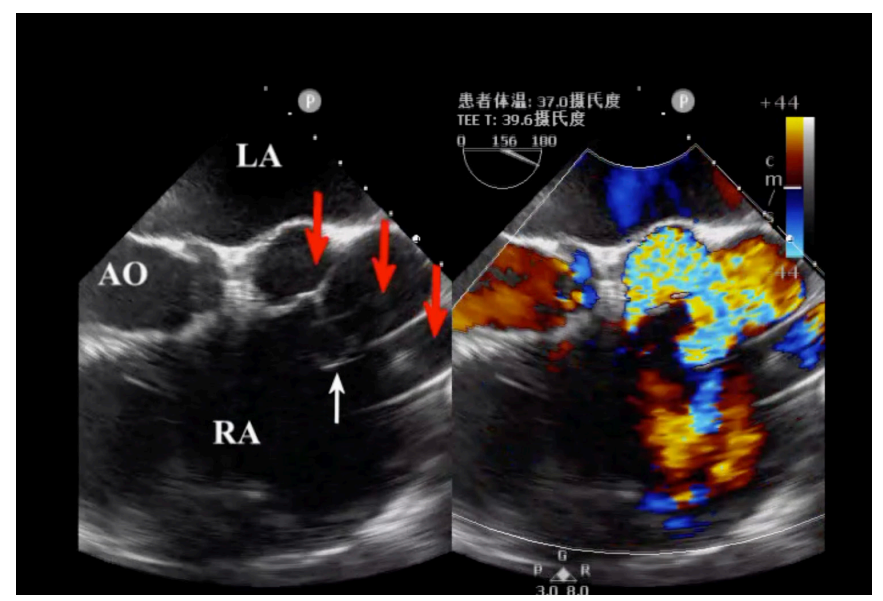

Video I. Transesophageal echocardiography. Transesophageal echocardiography revealed that the presence of a large right coronary artery (RCA) aneurysm with a fistula to the right atrium (RA).

https://vimeo.com/592913800

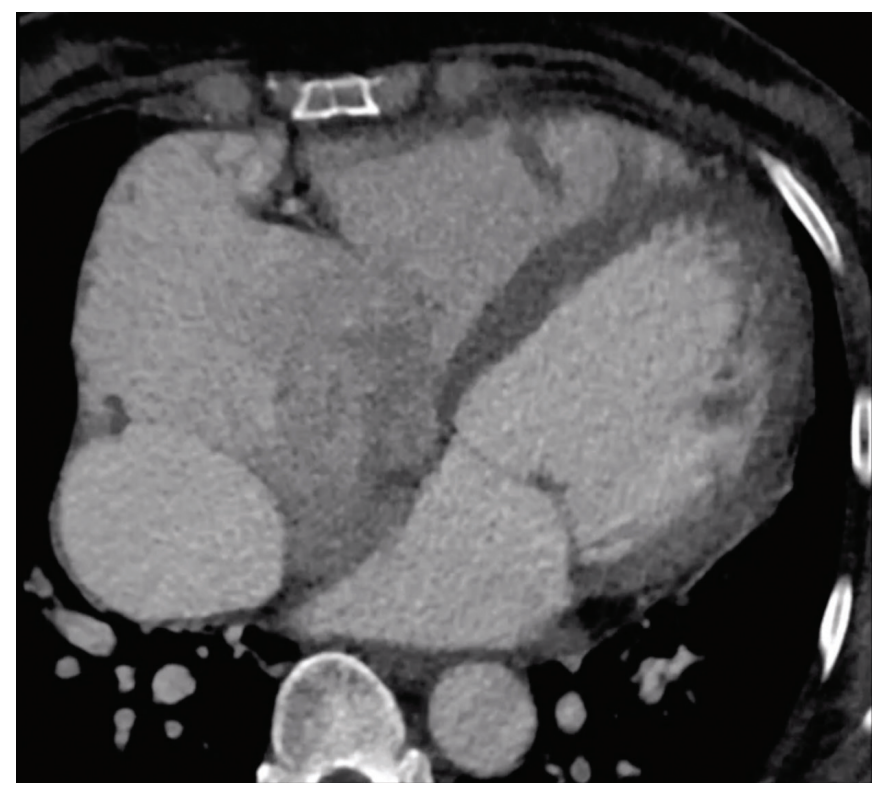

Video II. Preoperative cardiac computed tomography. Cardiac computed tomography demonstrated a giant coronary artery aneurysm that was on the right lateral surface of the right atrioventricular sulcus and formed a fistula to the RA.

https://vimeo.com/592914613

that the size of the fistula was $7 \mathrm{~mm}$ (Figure 1C and 1D and Video I in the Data Supplement). Cardiac computed tomography (CT) demonstrated a giant coronary artery aneurysm (CAA) $(63 \mathrm{~mm} \times 40 \mathrm{~mm})$ that was on the right lateral surface of the right atrioventricular sulcus and formed a fistula to the RA (Figure 1E and $1 \mathrm{~F}$ and Video II in the Data Supplement). Three-dimensional reconstruction clearly showed a giant atrial septal artery aneurysm with a fistula to the RA (Figure $1 \mathrm{G}$ and Video III in the Data Supplement). Coronary

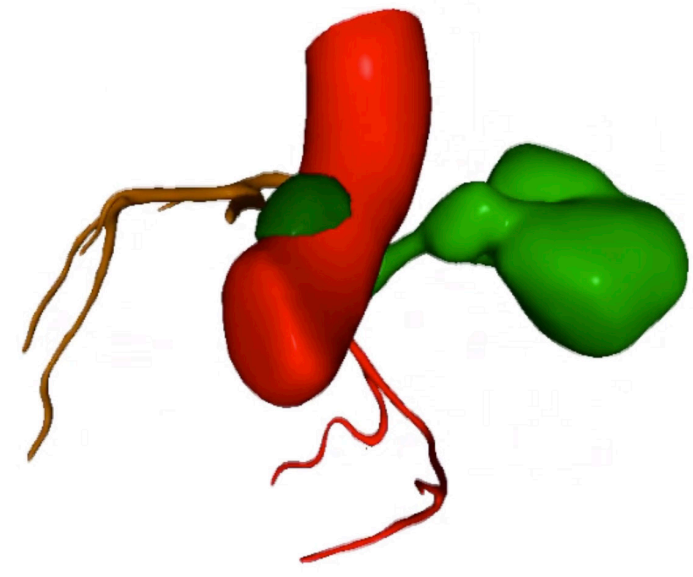

Video III. Three-dimensional reconstruction. Three-dimensional reconstruction clearly showed a giant atrial septal artery aneurysm with a fistula to the RA.

https://vimeo.com/592915555

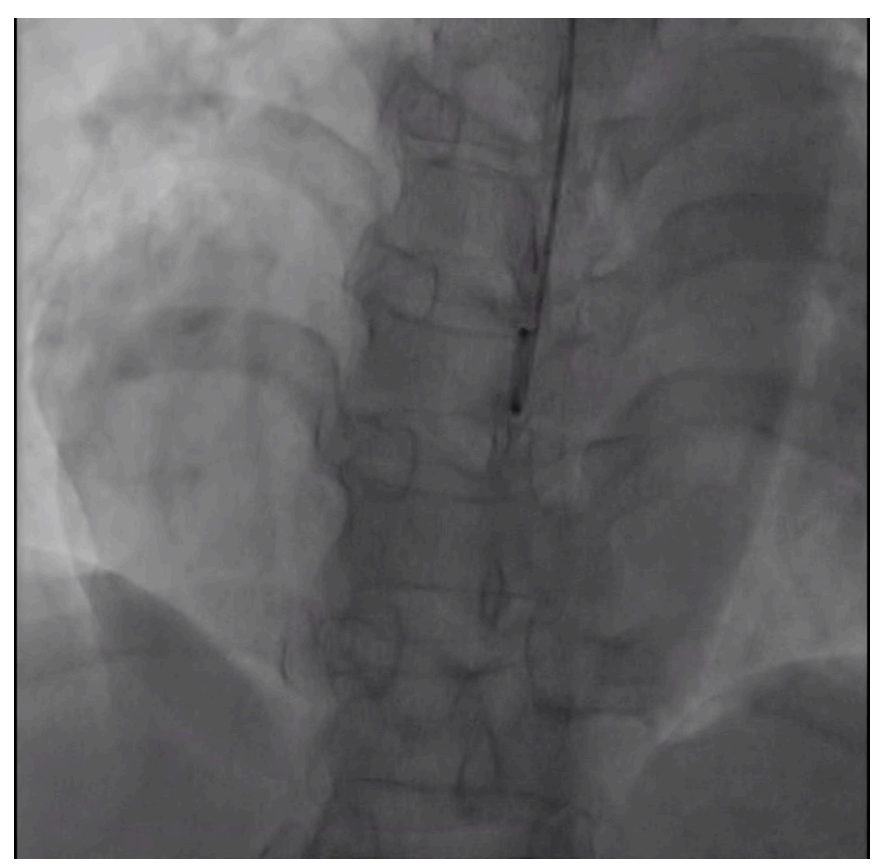

Video IV. Coronary angiography. Coronary angiography suggested that the proximal RCA was tortuous and dilated and confirmed the CAA and fistula.

https://vimeo.com/592916576

angiography suggested that the proximal RCA was tortuous and dilated and confirmed the CAA and fistula (Figure $1 \mathrm{H}$ and II and Video IV in the Data Supplement).

Cardiac computed tomography demonstrated successful resection of coronary artery aneurysm. According to 


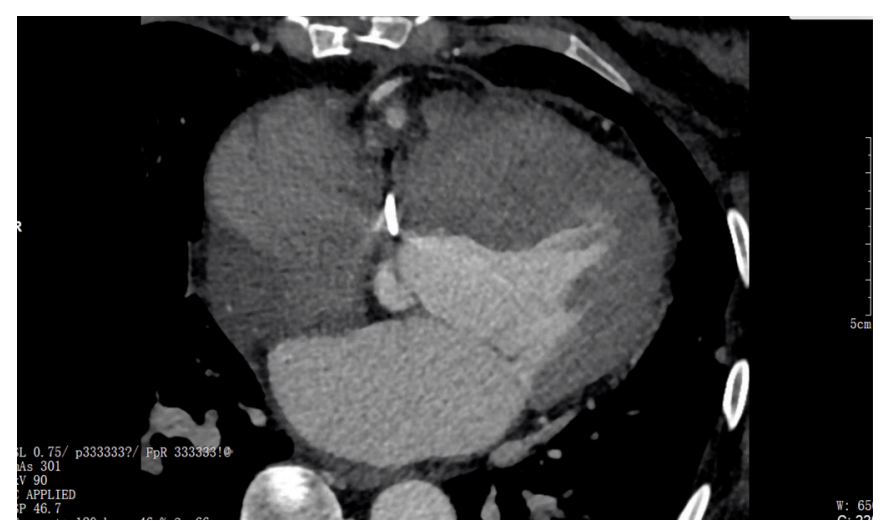

Video V. Postoperative cardiac computed tomography. https://vimeo.com/592917400

the results from the discussion among the multidisciplinary team, surgery was performed through a median sternotomy. We closed the aneurysm and fistula and performed tricuspid annuloplasty and the Maze procedure. A giant CAA (60 $\mathrm{mm} \times 60 \mathrm{~mm} \times 40 \mathrm{~mm}$ ) was located on the right lateral surface of the right atrioventricular sulcus. There was no thrombus inside the aneurysm. The fistula was identified as a $10-\mathrm{mm}$ hole at the lateral wall of the RA (Figure 1J and 1K). There was only one dilated atrial septal artery feeding the CAA through the atrial septum. First, the proximal atrial septal artery was knotted (Figure 1L). Then, the ostium of the fistula was closed with a bovine pericardium patch. The aortic root was perfused with cardioplegia, and the aneurysm did not expand. To reduce the risk of bleeding, the aneurysm wall was not removed. Finally, the Maze procedure (Med-Zenith, Beijing, China) and tricuspid annuloplasty with an Edwards 32-mm MC3 ring (Edwards Lifesciences, Inc., Irvine, CA, USA) were performed. The postoperative course was satisfactory. At 6-month follow up, cardiac computed tomography showed excellent effective of operation (Video $\mathrm{V}$ in the Data Supplement).

\section{DISCUSSION}

The coronary artery aneurysm is infrequent but not rare, accounting for about $0.35 \%$ of patients with cardiac catheterizations [Nunez-Gil 2020]. But, a giant coronary artery aneurysm $>20 \mathrm{~mm}$ in diameter is rare [Li 2005]. The most affected coronary artery is the RCA (40\%), left anterior descending (32\%), and left main being (3.5\%) [Syed 1997]. Patients suffering from giant coronary aneurysms located in the atrial septum with fistula formation to the RA are extremely rare [Osada 2016]. In the current article, we report a giant atrial septal artery aneurysm originating from a branch of the RCA combined with a coronary right atrial fistula. How did we confirm that the CAA originated from the atrial septal artery and not the sinus node branch artery? The artery originating from a branch of the RCA passed through the atrial septum, and the heart rate and rhythm after closing the artery were not obviously changed.
The causes of coronary artery aneurysms are atherosclerosis, Kawasaki disease, Takayasu arteritis, autoimmune diseases, trauma, coronary artery dissection, rheumatic, mycotic coronary emboli, syphilis, and so on [Ebina 2009]. Most CAAs may be completely asymptomatic and are only detected incidentally by coronary angiography or chest CT. The clinical symptoms included chest distress, palpitations, angina, dyspnea, sudden death, and so on. The main complications of CAA are as follows: local thrombosis formation within the aneurysm may cause distal embolization and myocardial infarction; aneurysm rupture can result in acute pericardial tamponade; giant CAA can lead to compression of adjacent structures [Kawsara 2018]; the fistula may bring about congestive heart failure and arrhythmias.

Before starting treatment, the CAA and the fistula should be evaluated using multiple diagnostic and imaging modalities [Wang 2020], such as echocardiography, coronary artery angiography, magnetic resonance imaging, and cardiac CT, especially three-dimensional reconstruction, which could help us to distinguish the physiological and anatomical characteristics of the CAA and fistula, such as the size, orientation, shape, location, number, thrombus, and hemodynamics. In the present case, although the CAA originated from an atrial branch of RCA and detailed information about the CAA and fistula was collected via these multiple imaging modalities before surgery.

The treatment strategies of CAA (medical, interventional, and surgical management) should be individualized based on the anatomical characteristics of the CAA and the patient's clinical characteristics. The optimal treatment is uncertain because of the lack of randomized controlled trials [Pham 2020]. However, operative therapy for giant CAA may be the ideal treatment. Surgical procedures include aneurysm ligation, resection, thrombectomy, or aneurysmectomy associated with bypass grafting if necessary [Kawsara 2018; Pham 2020]. Furthermore, surgical therapy also is the preferred option in the case of complications, such as fistula, embolism, compression, or rupture.

\section{ACKNOWLEDGEMENTS}

This study was supported by the Department of Science and Technology of Sichuan Province (2021YJ0208) and the Research Development Plan of the Affiliated Hospital of North Sichuan Medical College (2020ZD010, 2019BS002).

The authors are accountable for all aspects of the work in ensuring that questions related to the accuracy or integrity of any part of the work are appropriately investigated and resolved. Written informed consent was obtained from the patient for use data for academic publication purposes.

\section{REFERENCES}

Ebina T, Ishikawa Y, Uchida K, et al. 2009. A case of giant coronary artery aneurysm and literature review. J Cardiol 53(2):293-300.

Kawsara A, Núñez Gil IJ, Alqahtani F, et al. 2018. Management of Coronary Artery Aneurysms. JACC Cardiovasc Interv 9;11(13):1211-23. 
Li D, Wu Q, Sun L, et al. 2005. Surgical treatment of giant coronary artery aneurysm. J Thorac Cardiovasc Surg 130:817-21.

Nunez-Gil IJ, Cerrato E, Bollati M, et al. 2020. Coronary artery aneurysms, insights from the international coronary artery aneurysm registry (CAAR). Int J Cardiol 299:49-55.

Osada H, Kanemitsu N, Meshii K, et al. 2016. Giant atrial septal aneurysm originating from the right coronary artery. Interact Cardiovasc Thorac Surg 23(2):329-31.
Pham V, Hemptinne Q, Grinda JM, et al. 2020. Giant coronary aneurysms, from diagnosis to treatment: A literature review. Arch Cardiovasc Dis 113(1):59-69.

Syed M, Lesch M. 1997. Coronary artery aneurysm: a review. Prog Cardiovasc Dis. 1997 40(1):77-84

Wang H, Zheng Z, Mou Y. 2020. Giant Right Coronary Artery Aneurysm Resembling Cardiac Tumor: A Case Report. Heart Surg Forum 23(2):E165-E169. 